\title{
Pulsed light beams in vacuum with superluminal and negative group velocities
}

\author{
Miguel A. Porras \\ Departamento de Física Aplicada, ETSI Minas, Universidad Politécnica de Madrid, Rios Rosas 21, E-28003 Madrid, Spain \\ Isabel Gonzalo \\ Departamento de Óptica, Facultad de Físicas, Universidad Complutense de Madrid, Ciudad Universitaria s/n, E-28040 Madrid, Spain \\ Alessia Mondello \\ Dipartimento di Ingegneria Elettronica, Università degli Studi Roma Tre, Via della Vasca Navale 84, I-00146 Rome, Italy
}

(Received 26 February 2002; revised manuscript received 17 March 2003; published 17 June 2003)

\begin{abstract}
We study the group velocity of pulsed light beams in vacuum. Gouy's phase associated with the diffraction of transversally limited pulses can create a strong anomalous dispersion in vacuum leading to highly superluminal and negative group velocities. As a consequence, a focusing pulse can diverge beyond the focus before converging into it. The experimental feasibility is discussed.
\end{abstract}

DOI: 10.1103/PhysRevE.67.066604

PACS number(s): 42.65.Re, 42.65.Tg

\section{INTRODUCTION}

Propagation of light pulses at superluminal velocities has received a good deal of attention in recent years [1-5]. Superluminal behavior of light pulses has been predicted and experimentally demonstrated in evanescent modes of undersized waveguides [1], in Bessel $X$ waves propagating in free space [2], and in pulses traveling in transparent materials with pronounced enough anomalous dispersion [3], one of the most striking results being a negative group velocity of a temporally undeformable pulse, which in practice means that the pulse exits the dispersive material before entering [3].

In this paper, we show that arbitrarily high, even negative group velocities $v_{g}$ in temporally undeformable pulses are also possible in vacuum as an effect of the phase anomaly [6], or Gouy's phase associated with diffraction. As we shall see, a negative group velocity may result in a focusing pulse that appears to diverge from the focus before converging into it.

In vacuum, superluminal behavior has been previously described in Bessel $X$ pulses [2], and more recently in pulsed Gaussian beams $[7,8]$. In the former case, a superluminal light spot appears to move faster than $c$ (velocity of plane waves in free space), despite the subluminal value of $v_{g}$ of the Bessel $X$ pulse [9], as a geometrical effect similar to that of the scissors paradox [9].

In the case of pulsed Gaussian beams, it is the group velocity itself that may be superluminal under some circumstances $[7,8]$. A pulsed Gaussian beam propagates with $v_{g}$ $\lesssim c$ within the Rayleigh distance, but $v_{g} \gtrsim c$ beyond this distance [8]. On the contrary, $v_{g} \gtrsim c$ takes place within the Rayleigh distance if the pulsed Gaussian beam is ideally focused [7]. Though these results were obtained under the approximate paraxial theory of light beam propagation [10], they have been shown to remain unaltered and to be accurate when described from the nonparaxial vectorial KirchhoffSommerfeld diffraction formula for light beam propagation $[7,11]$; indeed superluminality is still stronger in the nonparaxial case [11].

The above results regarding superluminality in the vacuum group velocity are shown here to find a unified explanation in the dispersion induced by Gouy's phase, and moreover not to be exclusive to pulsed Gaussian beams.

The dependence of Gouy's phase shift with frequency acts as a dispersion that makes the phase and group velocities of a pulsed beam to be different and to differ from $c$. As shown in Sec. II, the dependence of Gouy's phase shift with frequency originates from the frequency-dependent nature of the diffraction phenomenon (identical transversal profiles of different frequencies diffract differently) and from the (possible) dependence of the transversal amplitude profile of a pulse with frequency. An expression for $v_{g}$ of arbitrary pulsed beams reflecting these two dependences is provided, and the conditions for subluminality, superluminality, and negative $v_{g}$ in terms of the pulse transversal profile and its dependence with frequency are established. Subluminality, superluminality, and negative $v_{g}$ due to the Gouy dispersion are shown to correspond to normal, anomalous, and strong enough anomalous dispersion in the propagation under an "equivalent" refraction index determined by the pulse transversal profile.

The different cases of slight superluminality previously studied in pulsed Gaussian beams, and some new ones for Gaussian and non-Gaussian pulsed beams, are then understood as different manifestations of the Gouy dispersion (Secs. III A and III B).

We also find (Sec. III C) that suitable optical elements, as radially graded mirrors $[12,13]$, can be used to introduce a suitable dependence of the transversal profile with frequency in the focusing pulse so as to enhance superluminality in $v_{g}$ up to arbitrarily high, even negative values around the focus. In Sec. V, we analyze in more detail the spatiotemporal features of a focusing pulsed Gaussian beam that propagates with negative $v_{g}$ in the vicinity of the focus, a pulsed Gaussian whose temporal form is, moreover, shown to remain nearly unchanged during propagation.

We point out that the superluminal group velocities described in this paper do not contradict Einstein's causality. The concept of group velocity makes only sense, strictly speaking, for the propagation of a pulse that does not expe- 
rience changes in its temporal form during propagation, and refers to the velocity of the pulse front, or geometrical locus of points where the pulse reaches its peak value at the same instant of time [6]. The time of pulse peaking at a front is determined by the relative phases of its monochromatic components, and changes from front to front because of their rephasing on propagation, rephasing that originates in our case from Gouy's phase. The front surface appears then to move at a velocity - the group velocity $v_{g}$ - which can take, in principle, an arbitrary value since different fronts are not, in general, causally connected. In particular, Gouy's rephasing can make, as shown below, a diverging front from the focus of a lens to occur at the same instant of time, even earlier than a converging one.

\section{GROUP VELOCITY, GOUY'S PHASE, AND INTENSITY CONCAVITY}

We consider a three-dimensional wave packet, or pulsed light beam $E(r, z, t), r=\sqrt{x^{2}+y^{2}}$, that results from the superposition

$$
E(r, z, t)=\frac{1}{\pi} \int_{0}^{\infty} d \omega \hat{E}_{\omega}(r, z) \exp (-i \omega t)
$$

of monochromatic light beams $\hat{E}_{\omega}(r, z)$ of different angular frequencies $\omega$.

All monochromatic components $\hat{E}_{\omega}(r, z)$ are assumed to be paraxial about the positive $z$ axis, and further to have, for simplicity, revolution symmetry around this axis. The slowly varying complex amplitude $\hat{\psi}_{\omega}(r, z)$ of the paraxial light beam $\hat{E}_{\omega}(r, z)\left[\hat{E}_{\omega}=\hat{\psi}_{\omega} \exp (i \omega z / c)\right]$ obeys then the paraxial wave equation

$$
\Delta_{\perp} \hat{\psi}_{\omega}+2 i \frac{\omega}{c} \frac{\partial \hat{\psi}_{\omega}}{\partial z}=0
$$

where $\Delta_{\perp}=\partial_{x x}+\partial_{y y}=(1 / r) \partial_{r}\left(r \partial_{r}\right)$ is the transversal Laplacian. Writing the slowly varying complex amplitude as $\hat{\psi}_{\omega}=a_{\omega} \exp \left(i \phi_{\omega}\right)$, i.e., in terms of its real amplitude $a_{\omega}(r, z)=\sqrt{I_{\omega}(r, z)}>0$ [squared root of intensity $I_{\omega}(r, z)$ ], and its phase $\phi_{\omega}(r, z)$, it is a straightforward calculation to obtain, from Eq. (2), the propagation equations for the phase and amplitude as

$$
\begin{aligned}
& \frac{\partial \phi_{\omega}}{\partial z}=\frac{c}{2 \omega}\left[\frac{\Delta_{\perp} a_{\omega}}{a_{\omega}}-\left(\frac{\partial \phi_{\omega}}{\partial r}\right)^{2}\right], \\
& \frac{\partial a_{\omega}}{\partial z}=\frac{c}{2 \omega}\left(a_{\omega} \Delta_{\perp} \phi_{\omega}+2 \frac{\partial \phi_{\omega}}{\partial r} \frac{\partial a_{\omega}}{\partial r}\right) .
\end{aligned}
$$

In particular, along the propagation axis $(r=0)$, and assuming that the light beam is smooth enough $\left[\left.\partial_{r} \phi_{\omega}\right|_{r=0}=0\right]$, Eq. (4) can be simplified to

$$
\frac{\partial \phi_{\omega}(z)}{\partial z}=-\frac{c}{2 \omega} C_{\omega}(z)
$$

where $\phi_{\omega}(z) \equiv \phi_{\omega}(0, z)$ is Gouy's phase of the monochromatic light beam $\hat{E}_{\omega}$, and

$$
C_{\omega}(z) \equiv-\frac{\left.\Delta_{\perp} a_{\omega}(r, z)\right|_{r=0}}{a_{\omega}(0, z)} .
$$

According to Eq. (5), Gouy's phase shift of a monochromatic light beam can be inferred from the property of its transversal intensity profile $C_{\omega}(z)$, an easily measurable quantity having an intuitive meaning: If the normalized intensity profile is expanded in power series about $r=0$, one readily obtains $I_{\omega}(r, z) / I_{\omega}(0, z)=1-(1 / 2) C_{\omega}(z) r^{2}+\cdots$. The parameter $C_{\omega}(z)$ thus determines the concavity of the intensity profile at $r=0$. An intensity profile $I_{\omega}(r, z)$ with a central maximum will have $C_{\omega}(z)>0$, and with a central hole, $C_{\omega}(z)<0$. For the Gaussian profile $a_{\omega}(r, z)$ $=\exp \left[-r^{2} / s_{\omega}^{2}(z)\right]$ of the Gaussian width $s_{\omega}(z)$, for instance, $C_{\omega}(z)=4 / s_{\omega}^{2}(z)>0$.

The dependence of Gouy's phase shift with the inverse of frequency in Eq. (5) reflects the frequency-dependent nature of diffraction. Gouy's phase shift may also depend on frequency if the concavities of the intensity profiles of the monochromatic components are different.

We are particularly interested in pulsed light beams $E(r, z, t)$ whose temporal form consists, on enveloped carrier oscillations, of a certain carrier frequency $\omega_{0}$. In this case the amplitude $a_{\omega}(r, z)$, regarded as a function of frequency, is a narrow function around $\omega_{0}\left(\Delta \omega / \omega_{0} \ll 1, \Delta \omega\right.$ being, e.g., the half-width at 1/e maximum amplitude). The phase velocity and group velocity at which the carrier oscillations and the envelope propagate in vacuum are given, respectively, by $[6]$

$$
\begin{aligned}
& v_{p}=\frac{\omega_{0}}{\left|\operatorname{grad}\left[\omega z / c+\phi_{\omega}(r, z)\right]_{\omega_{0}}\right|}, \\
& v_{g}=\frac{1}{\left|\operatorname{grad}\left[\omega z / c+\phi_{\omega}(r, z)\right]_{\omega_{0}}^{\prime}\right|},
\end{aligned}
$$

where the prime sign means differentiation with respect to angular frequency. In particular, along the propagation axis,

$$
\begin{aligned}
& \frac{v_{p}}{c}=\left[1+\frac{c}{\omega_{0}} \frac{\partial \phi_{\omega_{0}}(z)}{\partial z}\right]^{-1}, \\
& \frac{v_{g}}{c}=\left[1+c \frac{\partial \phi_{\omega_{0}}^{\prime}(z)}{\partial z}\right]^{-1} .
\end{aligned}
$$

It is then seen that the discrepancy of the on-axis value of the phase velocity $v_{p}$ from $c$ originates from Gouy's phase shift due to the transversal limitation of the wave. The discrepancy of the group velocity from $c$ arises from the frequency dependence of Gouy's phase, as discussed above. Negative group velocities (with the physical sense discussed by Wang et al. [3]) are, in principle, allowed by omitting the absolute value in the rigorous definition of group velocity [6]. From 
Eq. (5), the alternative expressions in terms of the intensity concavity can also be written as

$$
\begin{aligned}
& \frac{v_{p}}{c}=\left[1-\frac{c^{2}}{2 \omega_{0}^{2}} C_{\omega_{0}}(z)\right]^{-1}, \\
& \frac{v_{g}}{c}=\left\{1+\frac{c^{2}}{2 \omega_{0}^{2}}\left[C_{\omega_{0}}(z)-\omega_{0} C_{\omega_{0}}^{\prime}(z)\right]\right\}^{-1} .
\end{aligned}
$$

The values of $v_{p}$ and $v_{g}$ at a given cross section $z$ of a pulsed beam can be then predicted from the transversal profiles of the monochromatic light beam of frequency $\omega_{0}$ and neighboring frequencies at the same cross section $z$. The term $c^{2} C_{\omega_{0}}(z) / 2 \omega_{0}^{2}$ in Eq. (12) is present whenever there is a transversal profile, and originates from the frequency dependence of diffraction. The term $c^{2} C_{\omega_{0}}^{\prime} / 2 \omega_{0}$ contributes to the group velocity if, moreover, the transversal profile of the pulse depends on frequency. This dependence may be induced by diffraction itself upon propagation or suitably introduced by optical elements.

It is worthwhile to note that the above expressions of $v_{p}$ and $v_{g}$ can be formally obtained from the well-known formulas $v_{p} / c=n_{\omega_{0}}^{-1}$ and $v_{g} / c=\left(n_{\omega_{0}}+\omega_{0} n_{\omega_{0}}^{\prime}\right)^{-1}$ for the phase and group velocity of a plane pulse in a material medium if its refraction index is given by

$$
n_{\omega}(z)=1-\frac{c^{2}}{2 \omega^{2}} C_{\omega}(z)
$$

We can then say that a transversally limited pulse propagates in vacuum under the effects of diffraction as if the same pulse were propagating without transversal limitation in a material medium of refraction index $n_{\omega}$. It should then be possible, in principle, to design the transversal profile of a pulse in order to simulate its propagation in a material with a desired refraction index.

\section{SUBLUMINAL, SUPERLUMINAL, AND NEGATIVE GROUP VELOCITIES}

The group velocity of a pulsed light beam at a given cross section $z$ can be smaller or greater than $c$ depending on the relative values of the different terms in Eq. (12). From this equation, the condition of subluminal propagation $\left[v_{g}(z)\right.$ $<c]$ is found to be

$$
C_{\omega_{0}}^{\prime}(z)<C_{\omega_{0}}(z) / \omega_{0}
$$

and the condition of superluminality $\left[v_{g}(z)>c\right]$ is

$$
C_{\omega_{0}}^{\prime}(z)>C_{\omega_{0}}(z) / \omega_{0}
$$

Negative $v_{g}(z)$ can even take place if

$$
C_{\omega_{0}}^{\prime}(z)>C_{\omega_{0}}(z) / \omega_{0}+2 \omega_{0} / c^{2} .
$$

These conditions correspond to the usual conditions of subluminality $n_{\omega_{0}}^{\prime}>\left(1-n_{\omega_{0}}\right) / \omega_{0}, \quad$ superluminality $n_{\omega_{0}}^{\prime}<\left(1-n_{\omega_{0}}\right) / \omega_{0}$, and negative group velocity $n_{\omega_{0}}^{\prime}<$ $-n_{0} / \omega_{0}(<0)$ (strong enough anomalous dispersion) in terms of the refraction index, given in our case by Eq. (13).

In the following, we analyze different situations where subluminality and superluminality take place in the propagation of a pulsed beam. In particular, we show that the required variation of concavity with frequency imposed by condition (16) of negative group velocity can be generated in laboratory by the use of standard optics such as lenses and suitably designed filters.

\section{A. Subluminality}

A pulsed beam of the form $E(r, t)=P(t) b(r)$, for instance, having temporal form $P(t)$, and transversal amplitude profile $b(r)>0$ independent of frequency is nearly produced in the process of Kerr-lens space-time focusing, or by simply illuminating a graded neutral transparency with a plane pulse. The corresponding spectrum is $\hat{E}_{\omega}(r)$ $=\hat{P}_{\omega} b(r)$. Since the concavity is also independent of frequency, Eq. (12) leads to the group velocity

$$
\frac{v_{g}}{c}=\left[1+\frac{c^{2}}{2 \omega_{0}^{2}} C\right]^{-1},
$$

where $C=-\Delta_{\perp} b /\left.b\right|_{r=0}$. The group velocity is subluminal in the more frequent case of $C>0$. Subluminality originates from the dependence of Gouy's phase shift with frequency that arises from the frequency-dependent nature of diffraction. In the particular case of the pulsed Gaussian beam $P(t) \exp \left(-r^{2} / s^{2}\right)$, we obtain, as in Ref. [8], $v_{g} / c=(1$ $\left.+2 c^{2} / \omega_{0} s^{2}\right)^{-1}$.

\section{B. Superluminality}

Superluminality may occur in a variety of situations. If for instance, the on-axis concavity of the transversal profile $b(r)$ is instead $C<0$, the group velocity will be greater than $c$, as seen from Eq. (17).

Upon propagation, the initial pulsed beam $P(t) b(r)$ will acquire a diffraction-induced frequency dependence of the transversal amplitude profile. This fact leads, as noticed for pulsed Gaussian beams $[8,11]$, to superluminality at large enough propagation distances, regardless of the amplitude profile $b(r)$ and its concavity.

The pulsed beam spectrum $\hat{E}_{\omega}(r)=\hat{P}_{\omega} b(r)$ transforms on propagation up to the far field into

$$
\hat{E}_{\omega}(r, z)=\frac{\omega}{2 \pi i c z} \exp \left(\frac{\omega}{c} z\right) \exp \left(\frac{i \omega r^{2}}{2 c z}\right) \hat{P}_{\omega} \widetilde{b}\left(\frac{\omega r}{2 \pi c z}\right),
$$

where

$$
\widetilde{b}(\rho)=2 \pi \int_{0}^{\infty} d r r b(r) J_{0}(2 \pi \rho r)
$$


is the spatial two-dimensional Fourier transform of the radially symmetric function $b(r), J_{0}(\cdot)$ is the zero-order Bessel function of first class, and $\rho=\sqrt{\xi^{2}+\eta^{2}},(\xi, \eta)$ being conjugate variables of $(x, y)$. The spectrum amplitude is then $a_{\omega}(r, z)=(\omega / 2 \pi c z)\left|\hat{P}_{\omega}\right||\tilde{b}(\omega r / 2 \pi c z)|$, and concavity (6) at the far field,

$$
C_{\omega}(z)=-\left(\frac{\omega}{2 \pi c z}\right)^{2} \frac{\left.\tilde{\Delta_{\perp}} \tilde{b}(\rho)\right|_{\rho=0}}{\tilde{b}(0)},
$$

where $\widetilde{\Delta}_{\perp} \equiv \partial_{\xi \xi}+\partial_{\eta \eta}$. The elemental properties of the Fourier transformation lead to the relationship $\left.\widetilde{\Delta}_{\perp} \widetilde{b}(\rho)\right|_{\rho=0}$ / $\widetilde{b}(0)=-4 \pi^{2}\left\langle r^{2}\right\rangle$, where

$$
\left\langle r^{2}\right\rangle \equiv \frac{\int_{0}^{\infty} d r r^{3} b(r)}{\int_{0}^{\infty} d r r b(r)}
$$

is the squared rms width of the initial transversal amplitude profile $b(r)$. In conclusion, for each monochromatic component, the concavity of the amplitude profile at the far field is related to the rms width of the initial profile by the equation

$$
C_{\omega}(z)=\left(\frac{\omega}{c z}\right)^{2}\left\langle r^{2}\right\rangle .
$$

The proportionality of the concavity with $\omega^{2}$ makes condition (15) of superluminality to be satisfied. Indeed, from Eq. (12), the group velocity at the far field is given by

$$
\frac{v_{g}}{c}=\left(1-\frac{1}{2} \frac{\left\langle r^{2}\right\rangle}{z^{2}}\right)^{-1}>1 \text {. }
$$

As a third case of superluminality, we consider the focusing of the pulsed beam $P(t) b(r)$ of amplitude profile $b(r)$ independent of frequency at the entrance plane of a lens of focal length $f$. At the focal plane, the pulsed beam spectrum is given by Eq. (18) with the replacement of $f$ by $z$, as is well known. Proceeding as above, the concavity at the focal plane is $C_{\omega}=(\omega / c f)^{2}\left\langle r^{2}\right\rangle$, where $\left\langle r^{2}\right\rangle$ is the rms width on the lens. Consequently, the superluminal group velocity

$$
\frac{v_{g}}{c}=\left(1-\frac{1}{2} \frac{\left\langle r^{2}\right\rangle}{f^{2}}\right)^{-1}>1
$$

takes place at the focus. The Gaussian case $b(r)$ $=\exp \left(-r^{2} / s^{2}\right)$ in the limit of infinite Fresnel number $N_{\omega_{0}}$ $=s^{2} / \lambda_{\omega_{0}} f\left(\lambda_{\omega_{0}}=2 \pi c / \omega_{0}\right)$ was considered in Ref. [7]. We see, however, that the superluminality at the focus is independent of the Fresnel number and transversal amplitude profile.

\section{Strong superluminality}

Strong superluminality $\left(v_{g} \gg c, v_{g}<0\right)$ at the focus can be obtained if the transversal amplitude profile on the lens de- pends on frequency. In this case, the spectrum on the lens can still be conveniently written as $\hat{E}_{\omega}(r)=\hat{P}_{\omega} b_{\omega}(r)$, where $\hat{P}_{\omega}$ can be identified with the pulse spectrum at a typical point on the lens plane (e.g., at $r=0)$. The dependence of $b_{\omega}(r)$ with $\omega$ account for changes of the transversal amplitude profile with frequency. The concavity $C_{\omega}$ at the focus,

$$
C_{\omega}=\left(\frac{\omega}{c f}\right)^{2}\left\langle r^{2}\right\rangle_{\omega},
$$

depends now on frequency due to focusing $\left(\propto \omega^{2}\right)$ and to the possible variation of the rms width on the lens,

$$
\left\langle r^{2}\right\rangle_{\omega} \equiv \frac{\int_{0}^{\infty} d r r^{3} b_{\omega}(r)}{\int_{0}^{\infty} d r r b_{\omega}(r)}
$$

From Eq. (12), the group velocity at the focus is now given by

$$
\frac{v_{g}}{c}=\left(1-\frac{1}{2} \frac{\left\langle r^{2}\right\rangle_{\omega_{0}}}{f^{2}}-\frac{\omega_{0}}{2} \frac{\left\langle r^{2}\right\rangle_{\omega_{0}}^{\prime}}{f^{2}}\right)^{-1},
$$

which is (as in the previous case) independent of the exact shape of $b_{\omega}(r)$, and also of its possible distortions when widening with frequency. Superluminality is enhanced with respect to the case of $\omega$-independent profile if $\left\langle r^{2}\right\rangle_{\omega}$ at the lens grows with $\omega$ (i.e., $\left\langle r^{2}\right\rangle_{\omega_{0}}^{\prime}>0$ ).

In practice, growth of the width with frequency must be obtained by making use of the spatially dispersive properties of certain optical systems, as discussed in the following section. Assume for the moment that such a system is used to produce certain values of $\left\langle r^{2}\right\rangle_{\omega_{0}} \gg \lambda_{\omega_{0}}^{2}$ and $\left\langle r^{2}\right\rangle_{\omega_{0}}^{\prime}$ at the entrance plane of the lens. The focal length to be used to obtain $v_{g} \rightarrow \infty$ at the focus is, from Eq. (27),

$$
f^{2}=\frac{\left\langle r^{2}\right\rangle_{\omega_{0}}+\omega_{0}\left\langle r^{2}\right\rangle_{\omega_{0}}^{\prime}}{2} \quad\left(v_{g} \rightarrow \infty\right) .
$$

Slightly smaller $f$ will yield a negative group velocity. The focal length of Eq. (28) must, moreover, verify the condition of paraxiality $f \gg{\sqrt{\left\langle r^{2}\right\rangle}}_{\omega_{0}}$, or from Eq. (28),

$$
\left\langle r^{2}\right\rangle_{\omega_{0}}^{\prime} \gg\left\langle r^{2}\right\rangle_{\omega_{0}} / \omega_{0}
$$

which can be regarded as the condition for the optical system in order to obtain strong superluminality about the focus.

There is also a condition on the input pulse in order for our analysis to be valid. Equation (29) imposes an appreciable variation of the width on the lens plane with frequency. We must then ensure that not only the monochromatic component at $\omega_{0}$, but also at any $\omega$ within the frequency band $2 \Delta \omega$ of the pulse, focuses paraxially, i.e., $\lambda_{\omega} \ll \sqrt{\left\langle r^{2}\right\rangle} \ll f$ for all $\omega$ in $2 \Delta \omega$. If we assume, for simplicity, the approximate linear variation of the width $\sqrt{\left\langle r^{2}\right\rangle_{\omega}}$ 
$\simeq{\sqrt{\left\langle r^{2}\right\rangle_{\omega_{0}}}}+\sqrt{\left\langle r^{2}\right\rangle_{\omega_{0}}^{\prime}}\left(\omega-\omega_{0}\right)$ within $2 \Delta \omega$, we must then require $\sqrt{\left\langle r^{2}\right\rangle} \omega_{0}-\Delta \omega \gg \lambda_{\omega_{0}-\Delta \omega} \simeq \lambda_{\omega_{0}}$ for the smallest width in the spectrum, and $\sqrt{\left\langle r^{2}\right\rangle_{\omega_{0}}+\Delta \omega} \ll f$ for the largest one. These two conditions are seen to be satisfied if

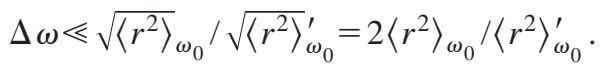

Equation (30) determines the maximum spectral bandwidth of the input pulse to achieve strong superluminality by paraxial focusing.

\section{IMPLEMENTATION OF STRONG SUPERLUMINALITY}

We have analyzed the possibility of achieving the above conditions of infinite group velocity at the focus of a lens by illuminating it with the output pulse from a graded mirror or transparency [12], as those made of a stack of dielectric layers, [13] and frequently used in laser resonators. Figure 1(a) shows an example.

The graded mirror is designed to produce a certain transversal amplitude profile $b_{\omega_{R}}(r)$ of a certain width $\left\langle r^{2}\right\rangle_{\omega_{R}}$ when illuminated with a specific reference frequency $\omega_{R}$. When illuminated with neighboring frequencies $\omega>\omega_{R}$, however, we have found that the produced amplitude profile $b_{\omega}(r)$ changes, and in particular that the spot size $\left\langle r^{2}\right\rangle_{\omega}$ grows significantly with frequency, as desired. Figure 1(b) shows the frequency-dependent width of the output amplitude profile from the multilayer, Fig. 1(a), when it is illuminated with monochromatic Gaussian profiles $\exp \left(-r^{2} / s_{0}^{2}\right)$ of different frequencies $\omega>\omega_{R}$ but constant width $s_{0}$. To obtain the output profile $b_{\omega}(r)$ for each illuminating frequency, we used the computer simulation program of Ref. [14]. The widths $\left\langle r^{2}\right\rangle_{\omega}$ were then calculated by numerical integration of Eq. (26).

If we select, for convenience, the frequency $\omega_{0}$ $=3.409 \mathrm{fs}^{-1}$, then $\left\langle r^{2}\right\rangle_{\omega_{0}}=0.17 \mathrm{~mm}^{2}$, and $\left\langle r^{2}\right\rangle_{\omega_{0}}^{\prime}$ $=1.645 \mathrm{~mm}^{2}$ fs. With these values, condition (29) for the usefulness of the graded mirror is loosely satisfied $\left(\left\langle r^{2}\right\rangle_{\omega_{0}}^{\prime}\right.$ $\left.\simeq 33\left\langle r^{2}\right\rangle_{\omega_{0}} / \omega_{0}\right)$. The focal length for $v_{g} \rightarrow \infty$ is indeed, from Eq. (28), $f=1.7 \mathrm{~mm} \gg \sqrt{\left\langle r^{2}\right\rangle_{\omega_{0}}}=0.41 \mathrm{~mm}$. Finally, condition (30) for the frequency band of the incoming pulse yields $\Delta \omega \ll 0.206 \mathrm{fs}^{-1}$, or equivalently, a pulse duration $\Delta t$ $\gg 10$ fs. In practice, the entire spectrum should lie within the operation range $\omega>\omega_{R}$ of the filter; we can then take, e.g., $\Delta \omega \leqslant 0.206 / 16$, which implies an input pulse duration $\Delta t$ $\geqslant 155$ fs.

\section{STRONG SUPERLUMINALITY IN FOCUSED PULSED GAUSSIAN BEAMS}

To investigate in more detail the effects associated with infinite or negative $v_{g}$, we have simulated the focusing of the pulsed beam $E(r, t)$ whose spectrum $\hat{E}_{\omega}(r)$ $=\hat{P}_{\omega} \exp \left(-r^{2} / s_{L, \omega}^{2}\right)$ has a frequency-dependent width $s_{L, \omega}$ on the lens plane. Using the standard rules of the Gaussian beam propagation [10], the spectrum amplitude at any propagation distance $z$ beyond the lens is given by

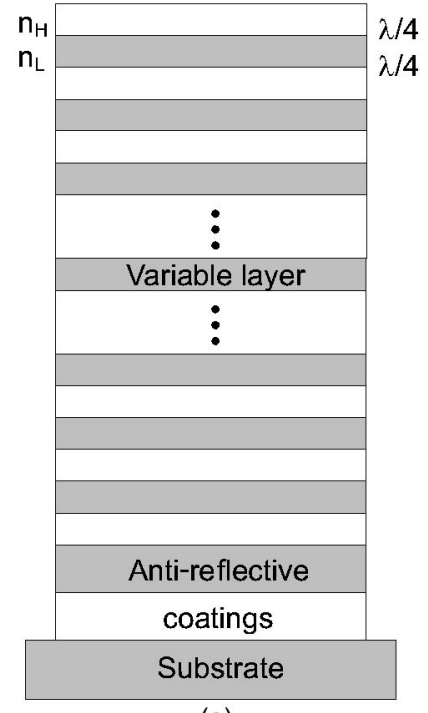

(a)

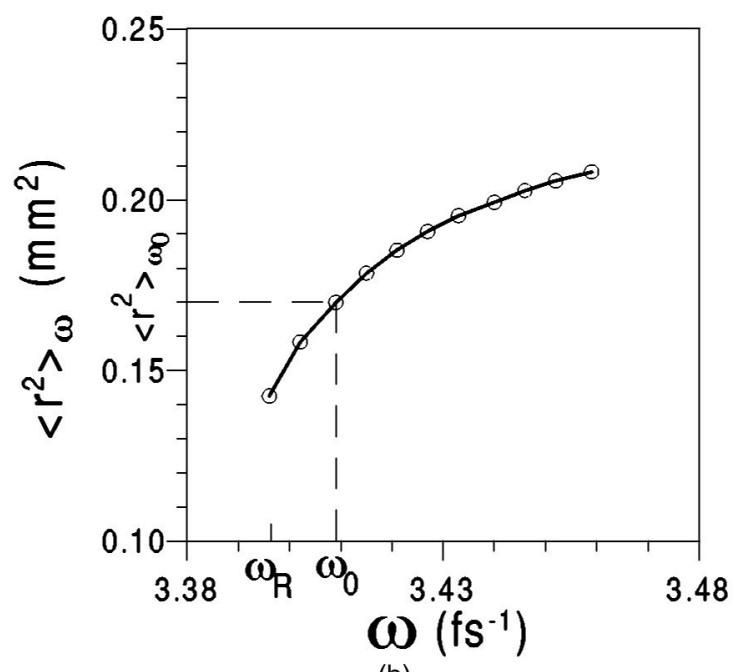

(b)

FIG. 1. (a) An all-dielectric multilayer high transmittance coating, obtained from a stack of quarter-wave $\left(\lambda_{\omega_{R}}=555 \mathrm{~nm}\right.$, or $\omega_{R}$ $\left.=3.396 \mathrm{fs}^{-1}\right)$ layers of alternate high $\left(n_{H}=2.20\right)$ and low index $\left(n_{L}=1.48\right)$ on a transparent substrate $\left(n_{S}=1.48\right)$, producing a radially variable transmittance. (b) The radius of the multilayer is chosen to be $r=10 \mathrm{~mm}$. We used the simulation computer program of Ref. [14] to obtain the radial transmittance curve $t_{\omega}(r)$ for $\omega_{R}$ and higher frequencies. When the multilayer is illuminated with the frequency-independent Gaussian profile $\exp \left(-r^{2} / s_{0}^{2}\right), s_{0}=0.5 \mathrm{~mm}$, the output is $t_{\omega}(r) \exp \left(-r^{2} / s_{0}^{2}\right)$, whose width is depicted (open circles). For convenience, we choose $\omega_{0}=3.409 \mathrm{fs}^{-1}$, for which $\left\langle r^{2}\right\rangle_{\omega_{0}}=0.17 \mathrm{~mm}^{2}$ and $\left\langle r^{2}\right\rangle_{\omega_{0}}^{\prime}=1.645 \mathrm{~mm}^{2} \mathrm{fs}$.

$$
a_{\omega}(r, z)=\hat{P}_{\omega} \frac{s_{L, \omega}}{s_{\omega}(z)} \exp \left[-\frac{r^{2}}{s_{\omega}^{2}(z)}\right],
$$

where

$$
s_{\omega}(z)=s_{L, \omega}\left[\left(1-\frac{z}{f}\right)^{2}+\left(\frac{2 c z}{\omega s_{L, \omega}^{2}}\right)^{2}\right]^{1 / 2}
$$



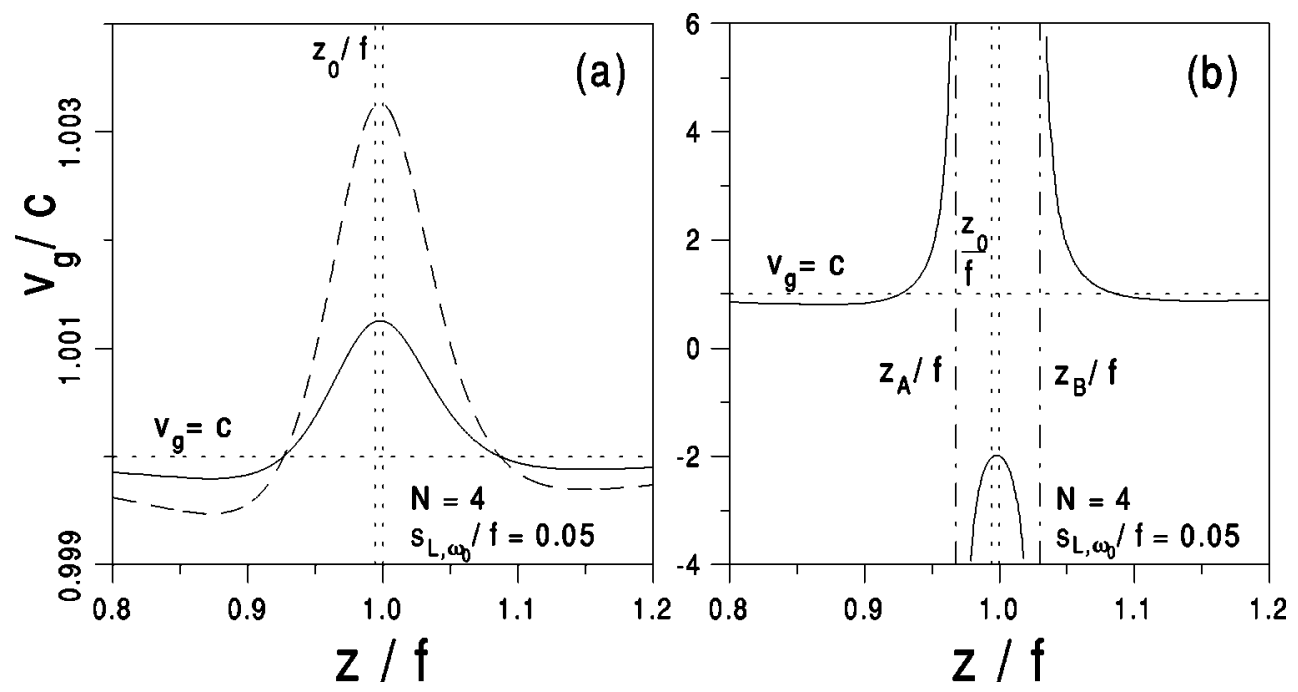

FIG. 2. Group velocity of focused Gaussian pulsed beams as functions of propagation distance from the lens. In all cases $N_{\omega_{0}}=4$, $s_{L, \omega_{0}} / f=0.05$. (a) $\omega_{0} s_{L, \omega_{0}}^{\prime} / s_{L, \omega_{0}}=0,0.003$, for the solid and dashed curves, respectively. (b) $\omega_{0} s_{L, \omega_{0}}^{\prime} / s_{L, \omega_{0}}=600$.

is the Gaussian width at distance $z$, and the spectrum phase is given by

$$
\phi_{\omega}(r, z)=-\tan ^{-1}\left[\pi N_{\omega} \frac{z-f}{z}\right]-\frac{\pi}{2}+\frac{\omega r^{2}}{2 c R_{\omega}(z)},
$$

where $N_{\omega}=s_{L, \omega}^{2} / \lambda_{\omega} f$ is the Gaussian Fresnel number, and

$$
\frac{1}{R_{\omega}(z)}=\frac{s_{L, \omega}}{s_{\omega}(z)}\left[\frac{1-z / f}{f}+\left(\frac{2 c}{\omega s_{L, \omega}}\right)^{2} z\right]
$$

The group velocity along the $z$ axis turns out to be, from Eq. (12),

$$
\frac{v_{g}(z)}{c}=\left\{1-\frac{s_{L, \omega_{0}}^{2}}{2 f^{2}} \sigma \frac{(1-\xi)^{2}\left[1-\left(\xi \pi N_{\omega_{0}}\right)^{2}\right]}{\left[1+\left(\xi \pi N_{\omega_{0}}\right)^{2}\right]^{2}}\right\}^{-1}
$$

where

$$
\sigma \equiv 1+2 \omega_{0} \frac{s_{L, \omega_{0}}^{\prime}}{s_{L, \omega_{0}}}
$$

and $\xi \equiv(z-f) / z$. At the focus $(\xi=0)$, Eq. (35) reduces to Eq. (27) with the identification $s_{L, \omega_{0}}^{2}=\left\langle r^{2}\right\rangle_{\omega_{0}}$. The curve $v_{g}(z)$ is shown in Fig. 2 for typical sets of parameters. The form of the curve is solely determined by $N_{\omega_{0}}$. A maximum
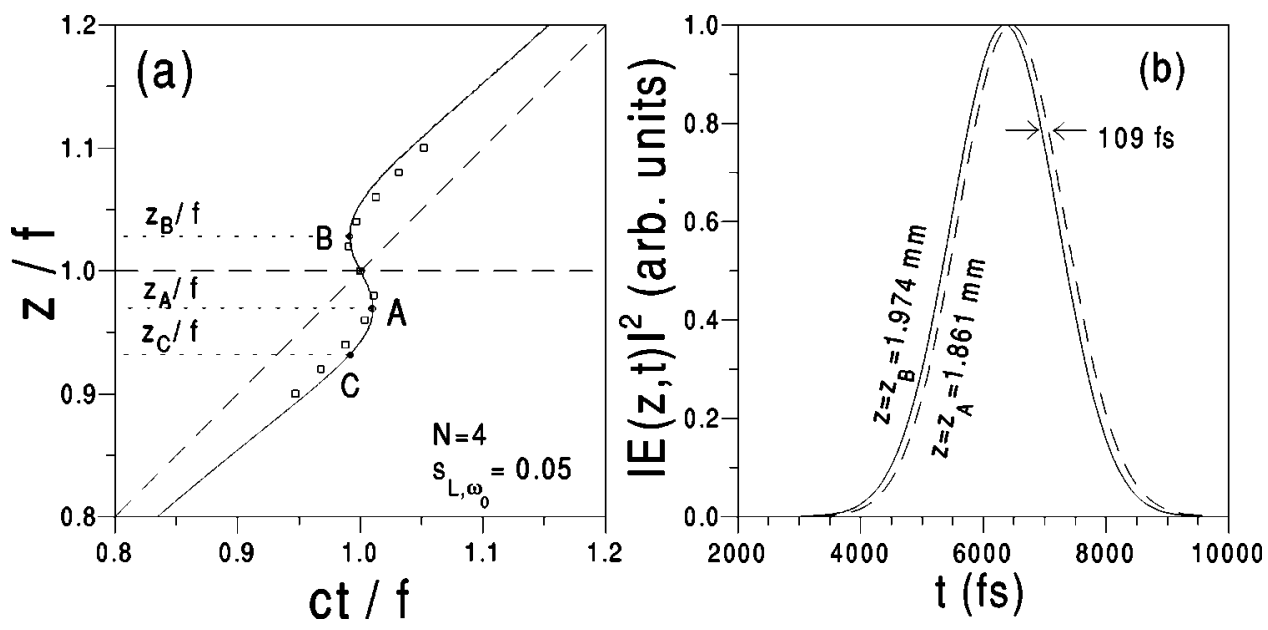

FIG. 3. (a) Solid curves: axial position of the pulse peak as a function of time, as predicted by $t=\left[\omega z / c+\phi_{\omega}(0, z)\right]_{\omega_{0}}^{\prime}$, where $\phi$ is the spectrum phase of the Gaussian beam [Eq. (33)], with $N_{\omega_{0}}=4, s_{L, \omega_{0}} / f=0.05$, and $\omega_{0} s_{L, \omega_{0}}^{\prime} / s_{L, \omega_{0}}=600$. Small squares: the same as solid line but numerically calculated from Eq. (1). (b) Normalized pulse temporal form at the indicated propagation distances from the lens of focal length $f=1.92 \mathrm{~mm}$, obtained from numerical integration of Eq. (1). The spectrum on the lens plane is $\hat{E}_{\omega}(r)=\hat{P}_{\omega} \exp \left(-r^{2} / s_{L, \omega}^{2}\right)$, with $\hat{P}_{\omega}$ $=\sqrt{\pi}(\Delta t / 2) \exp \left[-(\Delta t)^{2}\left(\omega-\omega_{0}\right)^{2} / 2\right], \omega_{0}=1.571 \mathrm{fs}^{-1}, \Delta \omega=1.309 \times 10^{-3} \mathrm{fs}^{-1}, s_{L, \omega_{0}}=0.096 \mathrm{~mm}$, and $s_{L, \omega_{0}}^{\prime}=36.67 \mathrm{~mm} \mathrm{fs}[$ to obtain, as in Figs. 2(b) and 2(a), $N_{\omega_{0}}=4, s_{L, \omega_{0}} / f=0.05$, and $\left.\omega_{0} s_{L, \omega_{0}}^{\prime} / s_{L, \omega_{0}}=600\right]$. 
is reached at an intermediate point between the focus $z=f$ and the waist position $z_{0}=f /\left(1+1 / \pi^{2} N_{\omega_{0}}^{2}\right)$ of the Gaussian beam with frequency $\omega_{0}$. If $N_{\omega_{0}}$ is large enough, the asymmetry of the curve $v_{g}(z)$ with respect to $z=f$ becomes inappreciable. The maximum enhances with increasing convergence angle $s_{L, \omega_{0}} / f$. Moreover, for given $s_{L, \omega_{0}} / f$ [as in the three curves in Figs. 2(a) and 2(b)], arbitrary enhancements are achieved by increasing $s_{L, \omega_{0}}^{\prime}$. For instance, in Fig. 2(b), $\omega_{0} s_{L, \omega_{0}}^{\prime} / s_{L, \omega_{0}}=600$ so that the left hand side of Eq. (28) is $2 / 3$ the right-hand side. An extended region with negative $v_{g}$ then appears around the focus. This region plays a similar role as the gas cell in the experiments of Ref. [3].

The behavior of a pulse with such an unusual group velocity is more easily understood from Fig. 3(a). The solid curve shows the time of arrival of the pulse peak (that travels at velocity $v_{g}$ ) at axial position $z$, as determined by the pulse front equation [6] $t=\left[\omega z / c+\phi_{\omega}(0, z)\right]_{\omega_{0}}^{\prime}$. The slope of the curve yields the group velocity in units of $c$. At $z_{A}$ and $z_{B}$, $v_{g}$ is infinite, and negative between them. As a consequence (as is apparent from the curve), the pulse peak may arrive earlier at axial points behind the focus than at points before the focus, e.g., earlier at $z_{B}$ than at $z_{A}$. The infinite group velocity at $z_{B}$ can be associated with the fact that the pulse peak appears to arrive instantly at $z_{B}$ from $z_{C}$. Indeed, in the time interval $\left(t_{C}, t_{A}\right)$, the pulse peak is at three positions at the same time.

Of course, the above interpretation for the behavior of a pulse with infinite and negative group velocities lies on the assumption that the pulse temporal form does not experience a significant deformation during propagation, in which case it is valid not only for the pulse peak but also for any temporal feature of the pulse. In Fig. 3(b), we have numerically verified that this is the case. The pulsed beam of spectrum on the lens $\hat{E}_{\omega}(r)=\hat{P}_{\omega} \exp \left(-r^{2} / s_{L, \omega}^{2}\right)$ with $\hat{P}_{\omega}=\sqrt{\pi}(\Delta t /$ 2) $\exp \left[-(\Delta t)^{2}\left(\omega-\omega_{0}\right)^{2} / 2\right]$ (i.e., $P(t)=\exp \left[-(t / \Delta t)^{2}\right]$ ) was propagated behind the lens according to the Gaussian beam rules [10], and the time-domain field was obtained from the numerical integration of Eq. (1), with $\omega_{0}=1.571 \mathrm{fs}^{-1}$, $s_{L, \omega_{0}}=0.096 \mathrm{~mm}, f=1.92 \mathrm{~mm}$, and $s_{L, \omega_{0}}^{\prime}=36.67 \mathrm{~mm}$ fs [to obtain, as in Figs. 2(b) and 3(a), the values $N_{\omega_{0}}=4$, $s_{L, \omega_{0}} / f=0.05$, and $\left.\omega_{0} s_{L, \omega_{0}}^{\prime} / s_{L, \omega_{0}}=600\right]$. Condition (30) is satisfied taking $\Delta \omega=1.309 \times 10^{-3} \mathrm{fs}^{-1}$, or $\Delta t=2 / \Delta \omega$ $=1528 \mathrm{fs}$. Figure $3(\mathrm{~b})$ shows the normalized pulse temporal form at $z_{A}=1.861 \mathrm{~mm}$ and $z_{B}=1.974 \mathrm{~mm}$ [points $A$ and $B$ of Fig. 3(a)]. There is no appreciable deformation in the pulse, which arrives at $z_{B}$ (after the focus) 109 fs earlier than at $z_{A}$ (before the focus). At other distances $z$, we have also observed the pulse form invariance. The time of arrival of the pulse peak at each selected $z$ is shown (small squares) in Fig. 3(a).

Further insight of the mechanism of pulse formation can be obtained from Fig. 4(a), in which the pulse axial form is plotted at selected increasing instants of time. The values of the parameters are the same as in Fig. 3(b). Nothing surprising can be seen in this figure, but a pulse that monotonically advances towards positive values of $z$ at the same time that

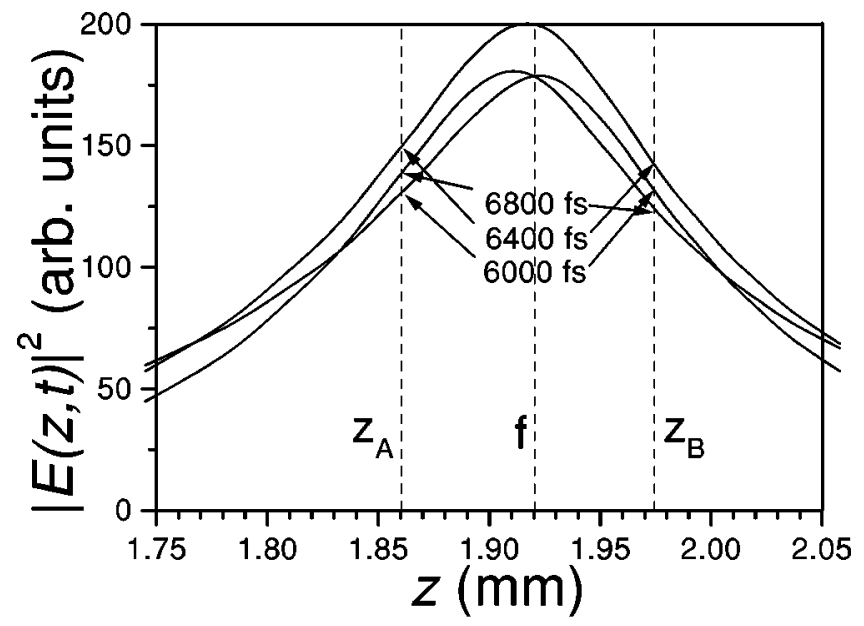

(a)

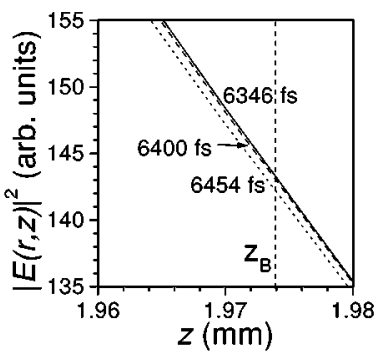

(b)

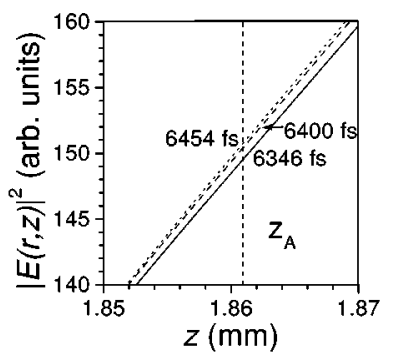

(c)
FIG. 4. (a) Axial form of the focused pulsed Gaussian beam at different instants of time. The values of the parameters are the same as in Fig. 3(b). (b) and (c) Axial form in the vicinity of $z_{B}$ and $z_{A}$ at different times about the corresponding peak times 6346 and 6454 fs, respectively.

slightly reshapes with increasing time $(6000,6400,6800 \mathrm{fs})$. The point to be understood is that axial reshaping is such that the time evolution at any fixed position $z$ is Gaussian of the same duration everywhere, and such that the Gaussian maximum occurs, e.g., earlier at $z_{B}$ after the focus than at $z_{A}$ before the focus, as can be seen in detail in Figs. 4(b) for $z_{B}$ (peak time $\sim 6346 \mathrm{fs}$ ) and 4 (c) for $z_{A}$ (peak time $\sim 6454 \mathrm{fs}$ ).

Figure 5 is the same as Fig. 4(a) but also shows the offaxis pulse structure. The joint effects of the overall pulse advancement towards positive values of $z$ and spatial pulse reshaping lead to the pulse front structure of Fig. 6. The pulse front, or geometrical locus of points where the pulse temporal form peaks at a same instant of time, can be calculated from the expression

$$
t=\left[\omega z / c+\phi_{\omega}(r, z)\right]_{\omega_{0}}^{\prime} .
$$

We see in Fig. 6 that when the convergent front advancing towards the focus intersects the axis at $z_{C}(6346 \mathrm{fs})$, a new elliptical front branch is born at position $z_{B}$ beyond the focus (curves 1), starting its motion with $v_{g}=\infty$. At $t=6400$ ( $1 \mathrm{fs}$ later than curve 3 ), the two branches join, transforming into a planar front that coincides with the focal plane backpropa- 

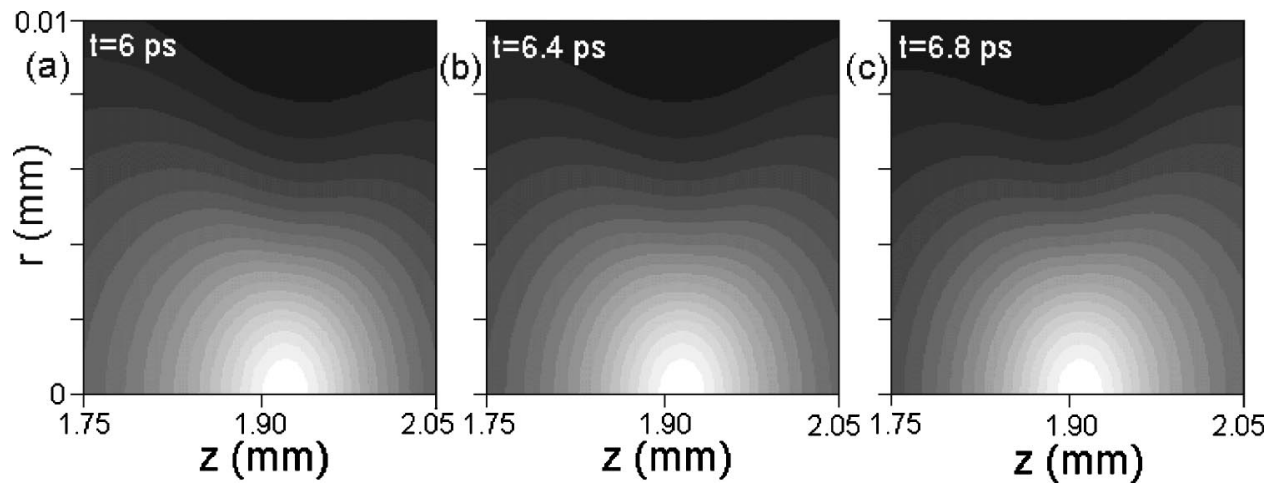

FIG. 5. Gray-scale plots of the spatial intensity distribution $|E(r, z, t)|^{2}$ of the focused pulsed Gaussian beam, at the same instants of time as in Fig. 4(a). The values of the parameters are the same as in Fig. 3(b).

gating at $v_{g} \simeq-2 c$, and a nearly elliptical front around the focus. At longer times (curves 4-6), the situation is nearly reversed; in particular, a branch of the front implodes at $z_{A}$ (6454 fs) with $v_{g}=\infty$. In short, the pulse peak diverges from the axial point $z_{B}>f$ before it reaches the focal plane. Indeed, the converging front never reaches this plane, but dies out at $z_{A}<f$.

\section{CONCLUSIONS}

We have studied the group velocity of light pulses with finite transversal size, which propagate in free space under the effects of diffraction. The on-axis group velocity differs from $c$ due to the Gouy dispersion, or dependence of Gouy's phase shift with frequency, a dispersion which originates from the frequency dependence of diffraction and from the possible dependence of the transversal profile with frequency, due to diffraction itself or introduced ad hoc. Simple criteria for the occurrence of subluminal, superluminal, and negative group velocities at a given cross section $z$ are established in Eqs. (14)-(16) in terms of the concavity of the pulse transversal profile $C_{\omega}(z)$ and its dependence with frequency about the carrier frequency. Then different typical situations where subluminality and superluminality take place have been described.

In particular, according to our analysis, it could be possible to observe experimentally large superluminal and negative group velocities in vacuum by focusing rather arbitrary pulses under the only condition that its spot size on the lens grows significantly with frequency. The required variation of spot size has been shown to be loosely attained with a multilayer graded mirror. Other devices are under study.

To finish, it appears necessary to stress that the described superluminal behavior refers to the group velocity, or velocity of the peak of the pulse, and not to the signal velocity. A pulse peak at a point of space appears when all monochromatic components are in phase. We have seen, in short, that adequate Gouy's rephasing can make pulse peaks to appear at different points at arbitrary relative times so that the group velocity, which refers to the "displacement" of the pulse peak, may take a rather arbitrary value.

\section{ACKNOWLEDGMENT}

The authors thank A. Piegari for helpful discussions.
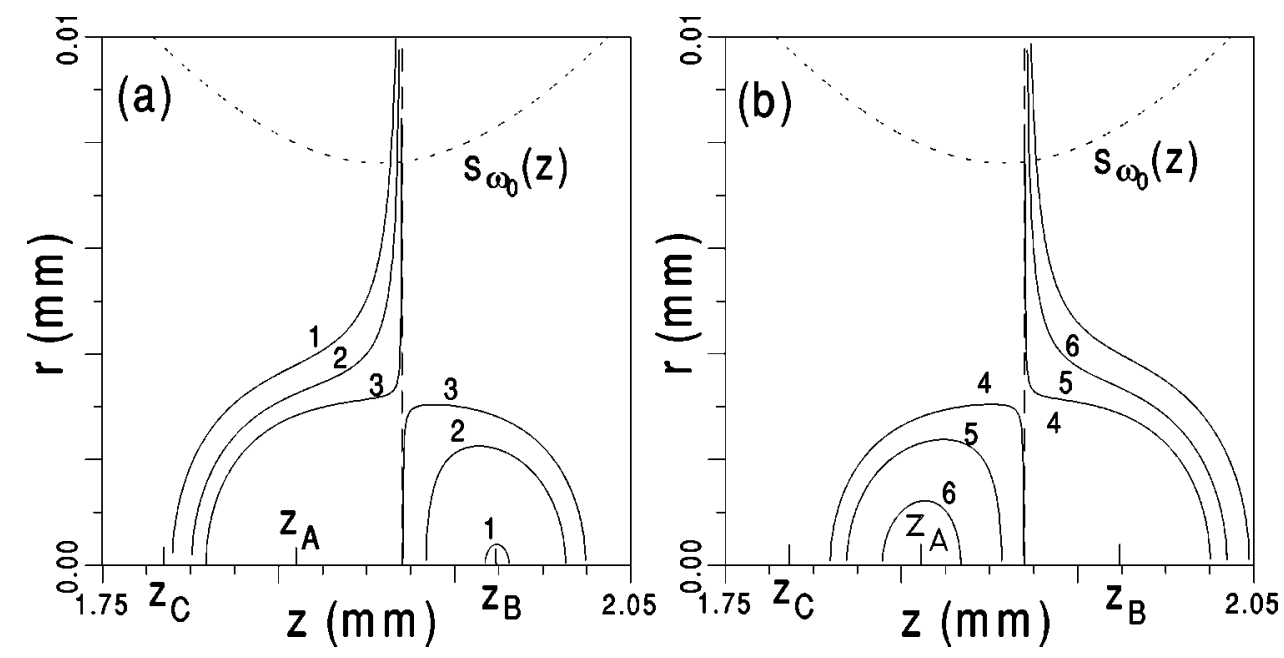

FIG. 6. Temporal evolution of the front surface, calculated from Eq. (37) at the instants of time (a) 1, $6346 \mathrm{fs} ; 2,6378 \mathrm{fs} ; 3,6399 \mathrm{fs}$. (b) 4, $6401 \mathrm{fs} ; 5,6422 \mathrm{fs} ; 6,6454 \mathrm{fs}$. 
[1] A.M. Steinberg, P.G. Kwiat, and R.Y. Chiao, Phys. Rev. Lett. 71, 708 (1993).

[2] P. Saari and K. Reivelt, Phys. Rev. Lett. 79, 4135 (1997).

[3] L.J. Wang, A. Kuzmich, and A. Dogariu, Nature (London) 406, 277 (2000).

[4] Y. Japha and G. Kurizki, Phys. Rev. A 53, 586 (1996).

[5] A. Dogariu, A. Kuzmich, H. Cao, and L.J. Wang, Opt. Express 8, 344 (2001).

[6] M. Born and E. Wolf, Principles of Optics (Cambridge University Press, Cambridge, 1999).

[7] Z.L. Horváth, J. Vinkó, Zs. Bor, and D. von der Linde, Appl. Phys. B: Lasers Opt. B 63, 481 (1996).
[8] M.A. Porras, Phys. Rev. E 65, 026606 (2002).

[9] P.W. Milonni, J. Phys. B: At. Mol. Opt. Phys. 35, R31 (2002).

[10] See for example, A.E. Siegman, Lasers (University Science Book, Mill Valley, CA, 1986).

[11] M.A. Porras, R. Borghi, and M. Santarsiero, Opt. Commun. 203, 183 (2002).

[12] For a review, see N.R. Belashenkov et al., J. Opt. Technol. 68, 320 (2001).

[13] A. Piegari, Appl. Opt. 35, 5509 (1996).

[14] In house computer program of the ENEA Thin Film Optics Laboratory, Casaccia, Rome, Italy. 